\title{
Kinetic and data-driven modeling of pancreatic $\beta$-cell central carbon metabolism and insulin secretion
}

\author{
Patrick E. Gelbach ${ }^{1}$, Dongqing Zheng ${ }^{2}$, Scott E. Fraser ${ }^{3}$, Kate White ${ }^{4}$, Nicholas A. Graham², and \\ Stacey D. Finley ${ }^{1,2,5^{*}}$
}

${ }^{1}$ Department of Biomedical Engineering, USC, Los Angeles, CA.

${ }^{2}$ Mork Family Department of Chemical Engineering and Materials Science, USC, Los Angeles, CA.

${ }^{3}$ Translational Imaging Center, University of Southern California, Los Angeles, California 90089, USA

${ }^{4}$ Departments of Biological Sciences and Chemistry, Bridge Institute, USC Michelson Center, USC, LoS

Angeles, CA.

${ }^{5}$ Department of Quantitative and Computational Biology, USC, Los Angeles, CA.

* Corresponding author

E-mail: sfinley@usc.edu 
Computational modeling of pancreatic $\beta$-cell metabolism

\section{Abstract}

Pancreatic $\beta$-cells respond to increased extracellular glucose levels by initiating a metabolic shift. That change in metabolism is part of the process of glucose-stimulated insulin secretion and is of particular interest in the context of diabetes. However, we do not fully understand how the coordinated changes in metabolic pathways and metabolite products influence insulin secretion. In this work, we apply systems biology approaches to develop a detailed kinetic model of the intracellular central carbon metabolic pathways in pancreatic $\beta$-cells upon stimulation with high levels of glucose. The model is calibrated to published metabolomics datasets for the INS1 823/13 cell line, accurately capturing the measured metabolite foldchanges. We first employed the calibrated mechanistic model to estimate the stimulated cell's fluxome. We then used the predicted network fluxes in a data-driven approach to build a partial least squares regression model. By developing the combined kinetic and data-driven modeling framework, we gain insights into the link between $\beta$-cell metabolism and glucose-stimulated insulin secretion. The combined modeling framework was used to predict the effects of common anti-diabetic pharmacological interventions on metabolite levels, flux through the metabolic network, and insulin secretion. Our simulations reveal targets that can be modulated to enhance insulin secretion. The model is a promising tool to contextualize and extend the usefulness of metabolomics data and to predict dynamics and metabolite levels that are difficult to measure in vitro. In addition, the modeling framework can be applied to identify, explain, and assess novel and clinically-relevant interventions that may be particularly valuable in diabetes treatment.

\section{Author Summary}

Diabetes is among the most common chronic illnesses, occurring when the $\beta$-cells in the pancreas are unable to produce enough insulin to properly manage the body's blood sugar levels. $\beta$-cells metabolize nutrients to produce energy needed for insulin secretion in response to high glucose, and there is a potential to harness $\beta$-cell metabolism for treating diabetes. However, $\beta$-cell metabolism is not fully characterized. We have developed a computational 
modeling framework to better understand the relationship between cellular metabolism and insulin production in the pancreatic $\beta$-cell. With this modeling framework, we are able to simulate metabolic perturbations, such as the knockdown of the activity of a metabolic enzyme, and predict the effect on the metabolic network and on insulin production. This work can therefore be applied to investigate, in a time- and cost-efficient manner, $\beta$-cell metabolism and predict effective therapies that target the cell's metabolic network.

\section{Introduction}

Pancreatic $\beta$-cells, the predominant cell type in the pancreatic islets of Langerhans, respond to and tightly regulate the body's blood glucose levels through insulin secretion. The process of glucose-stimulated insulin secretion (GSIS) is heavily dependent on the cells' intracellular metabolism[1,2]. Upon stimulation with high glucose levels, glucose is transported into the cell, causing an increase in glycolysis and oxidative phosphorylation, which lead to an increase in the cellular ATP/ADP ratio[3]. Increased ATP causes the closure of potassium $\left(\mathrm{K}^{+}\right)$channels and the opening of calcium $\left(\mathrm{Ca}^{2+}\right)$ channels, which promote the release of insulin. In order to accomplish this cascade of events, the $\beta$-cell has several key glucose-sensing metabolic steps that are widely considered to be vital to insulin secretion, including a specialized glucose transporter and the glucokinase reaction[4,5]. However, there are many additional pathways, metabolites, and reactions that are purported to be impactful in insulin secretion, depending on the context[6-10]. Additionally, the way coordinated changes in metabolic pathways and resulting metabolite pools influence insulin secretion is not fully understood. For that reason, there is a need to study pancreatic $\beta$-cell metabolism at a systems-level, identifying how sets of metabolic pathways work together to cause the observed biological properties of insulin-secreting pancreatic $\beta$-cells.

Given that appropriate secretion of insulin is vital to the successful maintenance of blood glucose homeostasis, an impaired metabolic state of the $\beta$-cell is closely linked to disease progression[11]. Lowered insulin secretion is correlated with the emergence and severity of Type 2 diabetes[12,13]. There is significant value in developing a deeper understanding of $\beta$-cell 
metabolic activity to determine underlying biological processes driving disease progression and to find novel potential mechanisms to treat the disease. Mass spectrometry-based metabolomics, which enables the quantitative measurements of cellular metabolites, has emerged as a way to analyze the cell's metabolic condition and thereby elucidate metabolic processes, in both healthy and diseased conditions. Because measurements of metabolite pool sizes often do not give insight into the cellular dynamics or kinetics, their utility may be extended by integrating the data into frameworks that predict transient dynamics[14]. Computational modeling of metabolism is one such technique that can leverage metabolomics data to predict dynamic behavior. Especially because the GSIS system is inherently timedependent, it is valuable to use nonlinear ordinary differential equation (ODE) models, trained and refined with metabolomics data, to further our understanding of the mechanisms driving insulin secretion.

Several mathematical models have been developed to understand the relationship between $\beta$ cell metabolism and insulin production. Topp et al. developed a simple three-equation model describing the relationships between glucose, insulin, and $\beta$-cell mass[15]. Modeling work by Bertram et el. focused primarily on ATP synthesis from pyruvate[16]. Magnus and Keizer studied the underlying mechanisms driving calcium cycling in $\beta$-cells, while Yugi and Tomita focused on mitochondrial metabolic activity[17-19]. Fridyland focused on the link between cellular metabolism and energetic processes such as the maintenance of a mitochondrial membrane potential[20]. Jiang et al. built upon many of these works to develop a detailed kinetic model of glucose-stimulated metabolism in $\beta$-cells[21]. However, the Jiang model lacked several glycolytic metabolites and pathways that may contribute to the activity of pancreatic $\beta$ cells, and it did not link insulin secretion to the modeled metabolic processes. Thus, while computational modeling has been used, there is currently no sufficient model of core central carbon metabolic pathways and how their dynamics correlate to insulin secretion.

To address this gap in knowledge, we develop a kinetic model of pancreatic $\beta$-cell intracellular metabolism, including key $\beta$-cell-specific metabolic pathways. We refine and train the model 
with published in vitro metabolomics data and assess the impact of metabolic perturbations on the entire network. We also pair predictions from the kinetic model with linear regression analysis to link metabolic processes with insulin secretion. Our integrative modeling approach is therefore a valuable tool to understand the dynamics of GSIS and may inform future research aimed at treating $\beta$-cell dysfunction.

\section{Materials and Methods}

MATLAB scripts for all analyses are available on GitHub:

https://github.com/FinleyLabUSC/PancreaticBetaCellMetabolism

\subsection{Model structure and metabolic pathways}

We constructed a kinetic model of the central carbon metabolism of the INS1 832/13 pancreatic $\beta$-cell line by building upon previously published models of intracellular metabolism

(Figure 1)[21-23]. The model consists of 56 metabolites and 61 enzyme-catalyzed metabolic reactions in five primary metabolic pathways: glycolysis, glutaminolysis, pentose phosphate pathway (PPP), the tricarboxylic acid (TCA) cycle, and the polyol pathway. These reactions occur in two cellular sub-compartments (cytosol and mitochondria). By including these central carbon metabolic pathways, the model is significantly more detailed and expansive than other published models of pancreatic $\beta$-cell metabolism. The model is represented as a series of nonlinear ODEs, characterized with Michaelis-Menten or bi-bi reaction kinetics, that describe how the concentrations of intracellular metabolites evolve over time in a pancreatic $\beta$-cell $[24,25]$. Thus, there is a single ODE for each metabolite included in the model. The ODEs are implemented in MATLAB and solved with the built-in ode15s differential equation solver[26] 


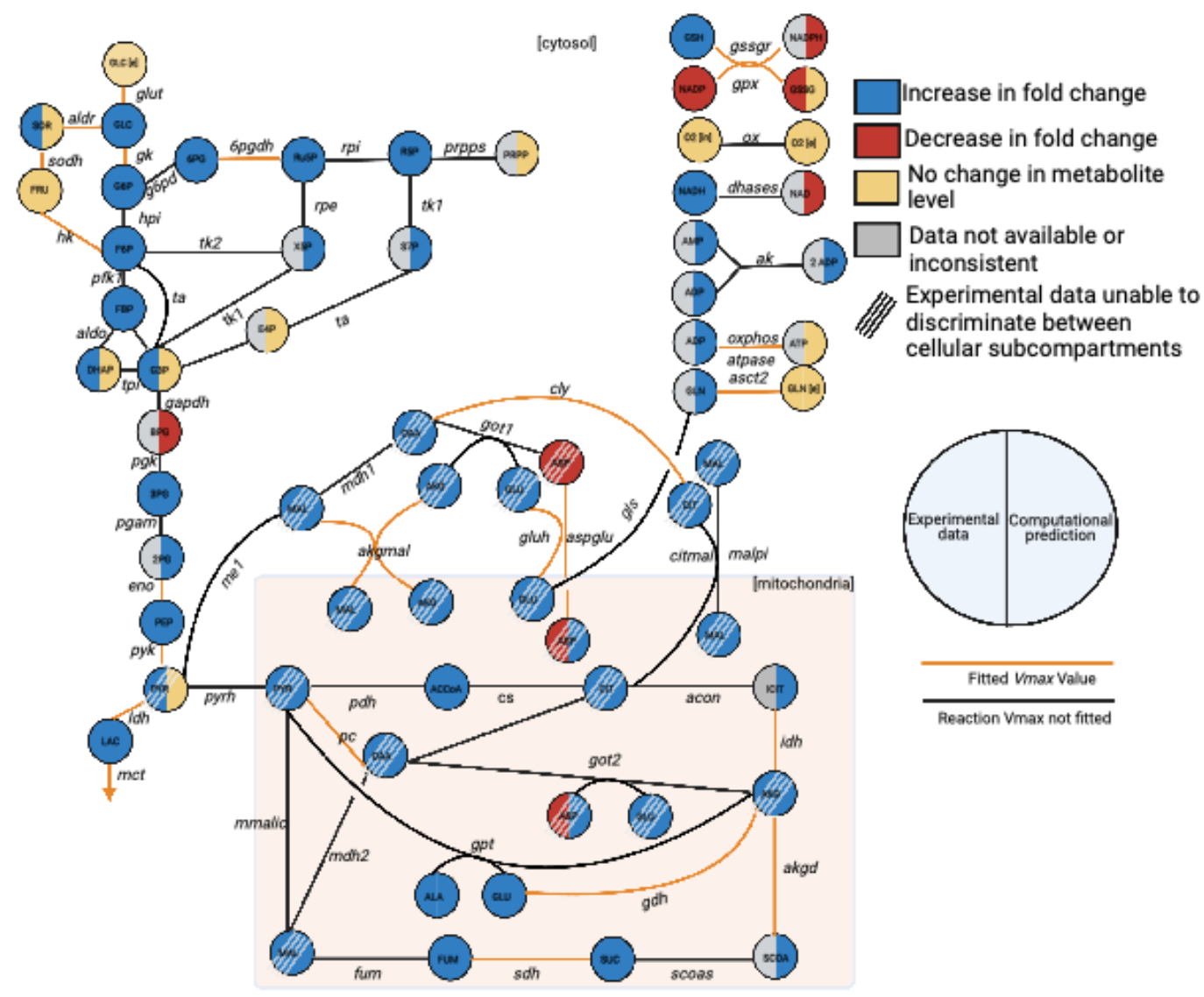

Figure 1: Metabolic network. The network of pancreatic beta cell central metabolism consists of 56 metabolites connected via 61 enzyme-catalyzed reactions, making up glycolysis, glutaminolysis, PPP, TCA Cycle, polyol pathways, and shuttles between the cytoplasm and mitochondria (denoted by the shaded rectangle). As part of model training, we performed a sensitivity analysis, and the reactions whose $V_{\max }$ values were significantly impactful are colored orange. We compared model predictions at the 60 -minute time point to qualitative shifts in metabolism as reported in the literature. The experimental observations are indicated by the left half of the metabolite nodes, and the model predictions are shown on the right. The model can differentiate between metabolite location (cytosol versus mitochondria), while the mass spectrometry approaches can only measure the total (pooled) amount of a metabolite; we have shaded those metabolites. The full set of abbreviations and reaction equations are given in the supplementary material.

\section{Glycolysis pathway}

Pancreatic $\beta$-cells respond to high blood glucose levels by metabolizing the extracellular glucose, triggering an increase in ATP production, which drives the closure of $\mathrm{K}^{+}$channels and the opening of $\mathrm{Ca}^{2+}$ channels, leading to the secretion of insulin[27]. The glycolytic pathway is therefore the primary pathway modulating insulin secretion, as it initiates the steps allowing for 
insulin release. The pathway begins with the GLUT2 glucose transporter, which, due to an estimated high Michaelis constant $\left(K_{\mathrm{m}}\right)$ value, acts as a glucose sensor[28]. Specifically, the rate of glucose uptake by the GLUT2 enzyme is proportional to extracellular glucose levels, thus modulating the glycolytic flux inside of the cell and the amount of insulin released[20]. $\beta$-cells also express the glucokinase ( $g k$ ) enzyme (called hexokinase type IV), which furthers contributes to the cells' sensitivity to glucose and acts as the rate-controlling step in GSIS[29]. The cells show low expression of the lactate dehydrogenase (Idh) enzyme and compensate for the need to manage NAD and NADH levels by increasing the activity of mitochondrial hydrogen shuttles, which are included in this model[30]. We also include downstream glycolytic intermediates, such as 2-phosphoglyceric acid (2PG), which have not previously been incorporated into kinetic models of pancreatic $\beta$-cell metabolism. In total, glycolysis is represented in the cytosol with 12 metabolites (2PG, 3PG, BPG, DHAP, F6P, FBP, G3P, G6P, GLC, LAC, PEP, PYR) and 13 reactions (aldo, eno, gapdh, gk, glut, hpi, ldh, mct, pfk1, pgam, pgk, pyk, and tpi).

\section{Pentose-phosphate pathway}

The relationship between the PPP and insulin release is not fully characterized, as it is relatively inactive in pancreatic $\beta$-cells[31]. However, the PPP contributes to the generation of NADPH, which is believed to influence or modulate insulin secretion[32]. Furthermore, PPP metabolites have been observed to increase in abundance following glucose stimulation[33-35]. We have therefore included the pathway in the model, with seven metabolites (6PG, E4P, PRPP, R5P, Ru5P, S7P, and X5P) involved in seven metabolic reactions (6pgdh, g6pd, prpps, rpi, ta, tk1, and $t k 2)$.

\section{Tricarboxylic acid cycle}

TCA intermediates have been linked with GSIS, as a majority of glucose is converted into pyruvate, which is then utilized in the TCA cycle. In particular, metabolic coupling factors (MCFs) such as glutamate are known to be linked with and amplify insulin secretion[36-39]. In addition, insulin secretion is likely dependent on cofactors such as NAD or NADP, which are 
produced through the TCA cycle[40-42]. The TCA cycle in this model is composed of 20 metabolites across two compartments. Thirteen are found in the mitochondria (ACCOA, ALA, AKG, ASP, CIT, FUM, GLU, ICIT, MAL, OAA, PYR, SCOA, and SUC) and seven are found in the cytosol (AKG, ASP, CIT, GLN, GLU, MAL, and OAA). Twenty-five metabolic reactions carry out the import and interconversion of those metabolites (acon, akgd, akgmal, asct2, aspglu, citmal, cly, cs, fum, gls, gluh, got1, got2, gpt, idh, malpi, mdh1, mdh2, me1, mmalic, pc, pdh, pyrh, scoas, and $s d h)$

\section{Polyol pathway}

The polyol pathway (also called the aldose reductase pathway) consists of the production of sorbitol from glucose in the aldose reductase reaction, and the subsequent conversion of sorbitol to fructose in the sorbitol dehydrogenase reaction[43]. The polyol pathway is relatively inactive in most physiological conditions due to the aldose reductase reaction's high $K_{\mathrm{m}}$ value and low affinity for glucose[44]. However, this pathway acts as a mechanism for the processing and disposition of glucose in hyperglycemic conditions, in order to protect against glucose toxicity $[45,46]$. The pathway is therefore of interest within the context of $\beta$-cell stimulation with high glucose levels, though it has never been included in existing models of $\beta$-cells. Additionally, there is substantial value in studying the polyol pathway within the context of the entire metabolic network being modeled, as the PPP reduces NADPH that is oxidized by the polyol pathway, leading to a potential metabolic cycle that may impact insulin secretion[33].

\subsection{Model equations and parameters}

The reactions in the metabolic pathways included in the model are simulated with enzymatic reaction rate expressions, and the majority of the model reaction rate laws and overall model structure are derived from Roy and Finley's model of pancreatic ductal adenocarcinoma[22]. $\beta$ cell-specific enzyme isoforms, such as glucokinase and the glucose transporter, are taken from the published models of pancreatic $\beta$-cells from Jiang and Fridyland[20,21]. Rate equations for the polyol pathway are taken from Cortassa et al., which simulates cardiac cell metabolism[23]. In total, the model contains 385 parameters, including 92 reaction velocities ( $V_{\max }$ values). To 
make the model specific to $\beta$-cell metabolism, we fit the $V_{\max }$ values to published metabolomics data obtained from the pancreatic $\beta$-cell line INS1 832/13 (described below).

\subsection{Initial conditions}

Where available, initial conditions were taken from published studies that quantified intracellular metabolite concentrations in $\beta$-cells[21]. The initial values for the metabolites for which there was no available data were set using Latin Hypercube Sampling[47]. We specified concentration ranges based on published measurements of metabolites in other cell types and sampled 50 sets of initial conditions[48,49]. The model was fit five times with each set of initial conditions, and we assessed the model agreement to data; the initial condition set that allowed for the best match to data was used for the subsequent fitting. This process limited the number of fitted model parameters to only the $V_{\max }$ values selected with subsequent sensitivity analyses (described in section 2.5), thereby avoiding overfitting.

\subsection{Data extraction}

There have been a range of published mass spectrometry-based experiments aimed at understanding the metabolic alterations that occur in INS1 832/13 pancreatic $\beta$-cells following stimulation with extracellular glucose. We compiled those studies and extracted the metabolite fold-changes using the internet-based webplotdigitizer tool[50]. The fold-change in insulin secretion amount was similarly calculated when available.

In the study published by Spegel et al., $\beta$-cells were treated with $2.8 \mathrm{mM}$ glucose for two hours and then $16.7 \mathrm{mM}$ glucose for 3, 6, 10, and 15 minutes[51]. Metabolite fold-change amounts were calculated for the $16.7 \mathrm{mM}$ glucose condition relative to the $2.8 \mathrm{mM}$ glucose condition for 14 metabolites: 2PG, 3PG, AKG, ALA, ASP, CIT, FUM, G3P, LAC, MAL, PEP, PYR, R5P, and SUC. Insulin was also measured for the same experimental conditions. We selected he 3- and 10minute time points as training data for use in model fitting. The 6- and 15-minute time points were withheld from model parameter estimation in order to be used for validation. Some of the measured metabolites are found in both the cytosol and mitochondria; however, they cannot 
be distinguished via metabolomic analysis. Therefore, we fit the measured fold-change of the total metabolite pool.

In the work published by Malmgren et al., $\beta$-cells were treated with $2.8 \mathrm{mM}$ or $16.7 \mathrm{mM}$ glucose for 60 minutes[52]. Fold-changes were calculated for 14 metabolites: AKG, ALA, ASP, CIT, FUM, G3P, G6P, GLC, GLU, ICIT, LAC, MAL, PYR, and SUC. All of these measurements were used as training data.

In addition to the quantitative data sets listed above, data from eleven other published papers were used as qualitative validation of predicted fold-change direction for metabolites in the model upon treatment with above-basal glucose levels[33-35,51-60], shown in Figure S1.

\subsection{Parameter estimation}

We first performed a pairwise parameter identifiability analysis and found 11 forward or reverse reaction velocities ( $V_{\mathrm{f}}$ or $V_{\mathrm{r}}$ parameters) that were found to be correlated to other model parameters. Therefore, we defined these reaction velocities using equilibrium constants. That is, we set the reverse reaction velocity, $V_{R}$, to be expressed in terms of the forward reaction velocity and the equilibrium constant, $K$, so that $V_{\mathrm{r}}=V_{\mathrm{f}} / K$. We therefore had $81 V_{\max }$ parameters available for model fitting and subsequent analyses.

Next, we identified the parameters that should be fit to the training data. The extended Fourier Amplitude Sensitivity Test (eFAST), a variance-based global sensitivity analysis method, was performed by simulating the same in vitro experimental methods used to collect the metabolomic data[61]. The eFAST method varies model inputs (the $81 V_{\max }$ parameters) two orders of magnitude above and below their baseline values in order to understand the sensitivity of the model outputs (the metabolite fold-changes). We can therefore identify the most influential model parameters to fit to experimental data, so that the model can accurately match the data without overfitting. Based on the eFAST results, we identified 32 influential model parameters by selecting the parameters that had sensitivity indices above 0.85 . 
Parameter estimation was then performed via particle swarm optimization (PSO) with the Parameter Estimation Toolbox (PESTO) in MATLAB (Mathworks, Inc.), minimizing the weighted sum of squared residuals (WSSR)[62,63]. In order to fit the data, we computationally simulated the experimental protocols for each dataset described in Section 2.4. PSO is a stochastic global optimization tool that iteratively updates sets of randomly seeded particles (parameter sets) to converge upon a single parameter set that minimizes the WSSR. The WSSR error calculation is similar to the sum of squared residuals (SSR), but each data point is weighted by its associated standard deviation and magnitude. In the PSO protocol, each parameter is allowed to vary 100 times above and below its initial estimate, and each fitting run carries out 2,000 steps; this limits the computational cost while exploring the total parameter space. One hundred fitting runs were performed with PSO, and the best parameter sets were selected (a total of 8 best fits) based on the WSSR. These best fitted parameter values were used for all subsequent analyses and figures.

\subsection{Partial least squares regression}

Using the trained kinetic model, we predicted the flux through each of the 61 metabolic reactions over time in 1-minute intervals. We then found the average flux through each reaction over the time period for which we had metabolomics data $(3,6,10,15$, or 60 minutes). Thus, we predicted a single time-averaged flux value for a particular length of glucose stimulation. The predicted flux values are inputs to a regression analysis, and the corresponding fold-change amounts for insulin secretion for INS1 832/13 cells stimulated with $16.7 \mathrm{mM}$ glucose relative to insulin secretion following $2.8 \mathrm{mM}$ glucose from the published studies are outputs of the regression analysis.

We performed partial least squares regression (PLSR), a multivariate dimensionality reduction technique that seeks to determine a mathematical correlation between a chosen input vector with an output measurement of interest[64]. PLSR produces the components, weighted linear combinations of the inputs, that correlate with the output, and we used the SIMPLS algorithm 
to complete this analysis[65]. The input matrix was 5 rows by 61 columns, with the columns representing the predicted average flux for each model reaction, and the rows representing the five time points of interest. The output matrix was 5 rows by 1 column, corresponding to experimentally measured insulin secretion fold-change at the five time points. By performing partial least squares regression, we specify the relationship between flow of material through the metabolic network and insulin released by the $\beta$-cell. The 6 - and 10 -minute time points were withheld to be used as validation data, while the $3-, 15-$, and 60-minute time points were used to build the PLSR model. This process was performed where the input matrix was predicted using each of the eight best-fit parameter sets. Thus, all predictions from PLSR analysis are showing the averaged results across the eight PLSR models. We evaluated the PLSR model fitness with the $R^{2}$ and $Q^{2} Y$ values, which range from 0 to 1 . The $R^{2}$ value indicates model agreement to the training data, termed "goodness of fit", and the $Q^{2} Y$ value assesses the ability of the model to predict data not used for training, i.e., "goodness of prediction"[66].

We use two quantities calculated in the PLSR analysis to gain biological insight into how intracellular metabolism influences insulin secretion. The PLSR analysis estimates the variable importance of projection (VIP) score for each reaction flux. The VIP score quantifies the contribution of each input value to the model predictions. Here, the VIP score identifies the metabolic reactions that are most strongly correlated to insulin secretion. Generally, VIP scores greater than one indicate influential inputs[67]. In addition, by assessing the PLSR model weights, we determined the effect that altering flux through the metabolic network would have on insulin secretion. For example, a negative weight indicates that increasing flux through that reaction would decrease insulin secretion.

We also performed PLSR analysis to determine how intermediate time points influence insulin secretion, to understand the time-dependent nature of the link between metabolism and insulin at a higher resolution. In order to accomplish this goal, we again used the metabolic network's fluxome predicted by the fitted kinetic model of metabolism as an input to a PLSR model; but this time, at 1-minute intervals for a single time course. For example, the kinetic 
model predicted the fluxes through each reaction for the first three minutes of simulation and used this set of three predicted fluxes as inputs to a PLSR model. The fold-change in the secreted insulin calculated from the experimental data was used as the PLSR output. Because the PLSR approach requires multiple data points for both its input and output, we assumed a linear increase in secreted insulin per minute, thus giving the same number of outputs (foldchange of insulin secretion calculated per minute from the experimental data) as inputs (predicted reaction fluxes for each minute). We performed this analysis to determine the timedependent relationships between reaction fluxes and insulin secretion over 3, 6, 10, and 15 minutes. As described above, we used the VIP scores and weights from the PLSR models to identify the most important reaction fluxes for each one-minute interval within each time course.

\subsection{Kinetic model perturbations}

Variation of each model parameter. The kinetic network can be used to predict the effects of metabolic perturbations on metabolite levels and the fluxes through the metabolic reactions, thereby giving a prediction of the systems-level response of the cell. Using the fitted kinetic model, we simulated metabolic perturbations, where we increased and decreased the rate of each model reaction by a factor of two and assessed the impact of that perturbation on all modeled metabolites, compared to the unperturbed baseline condition.

Implementation of pharmacological interventions. We selected three anti-diabetic pharmacological interventions to simulate in the model. Two perturbations are based on existing agents: metformin, which is the most common drug taken by diabetic patients but whose impact on $\beta$-cells is not fully understood and agrimony, a medicinal plant believed to act as an antioxidant in the $\beta$-cell. Lastly, we simulated the upregulation of the adenylate kinase (ak) reaction, which reversibly catalyzes ATP and AMP from two ADP molecules, as that intervention was predicted to be the most beneficial for increasing insulin secretion according to the PLSR model. 
Metformin primarily acts on the peripheral tissues and organs by reducing hepatic glucose production and increasing skeletal muscle glucose uptake. Together, these effects reduce hyperglycemia and effectively treat diabetes[68]. It is unclear how or if metformin affects pancreatic $\beta$-cells in vivo. Lamontagne et al. proposed that metformin drove "metabolic deceleration", wherein the INS1 $\beta$-cell experiences a decrease in glucose-induced insulin secretion, thereby protecting the cells from hyper-responsiveness or hyperglycemic glucotoxicity and lipotoxicity[69]. Others have shown that metformin protects against $\beta$-cell exhaustion by reducing the body's blood glucose levels[70-72]. To test this hypothesis, we decreased glucose transport into the cell by reducing the $V_{\max }$ value for the glut reaction by $80 \%$. We then calculated the effect on metabolites, metabolic fluxes, and insulin secretion, and compared model predictions to reported metabolomics data when possible.

Agrimony (Agrimony eupatoria) is a medicinal plant used around the world to treat diabetes, especially in traditional Eastern medicine practices[73,74]. It has been shown to affect insulin secretory activity, both in patients and in a pancreatic $\beta$-cell line in vitro[75]. It is believed that agrimony acts as an antioxidant in the $\beta$-cell[76]. It is well known that oxidative stress, induced by reactive oxygen species (ROS) and reactive nitrogen species (RNS), impairs $\beta$-cell activity and is a contributing factor observed in diabetes progression[77-79]. The PPP is believed to impact the oxidative stress response, as it is a one of the primary NADPH-generating pathways. The generation of NADPH in high-glucose conditions is shown to reduce cell inflammation[80,81]. Furthermore, an increase in PPP flux shifts the cell metabolism away from ROS-generating pathways, limiting further stress on the cells[82]. To simulate the action of agrimony, we perturbed the glucose-6-phosphate dehydrogenase $(g 6 p d)$ reaction with a five-fold increase of its $V_{\max }$ value, to simulate overexpression, as $g 6 p d$ is the primary upstream controller of PPP activity[83-85].

Finally, based on results from PLSR modeling, we simulated the effects of perturbing the $a k$ reaction. We implemented a five-fold increase in $V_{\max }$ value and assessed the effect on the metabolic network as a whole. 


\section{Results}

We developed a kinetic model capable of simulating the dynamics of intracellular metabolism of the pancreatic $\beta$-cell. The model includes key metabolites and reactions involved in glycolysis, glutaminolysis, PPP, TCA cycle, and polyol pathways, with cytosolic and mitochondrial compartments. The model consists of 56 metabolites and 61 metabolic reactions and was trained to published mass spectrometry-based metabolomics data sets. The fitted model predictions were used to create a PLSR model to study the relationship between flux through the metabolic network and secreted insulin. We then assessed the impact of various metabolic perturbations on model predictions.

\subsection{Model fitting to training and validation metabolomics data}

We developed the model structure to comprise pancreatic $\beta$-cell central carbon metabolism, including isoforms and pathways unique to the $\beta$-cell. After performing a parameter identifiability analysis to exclude the forward and reverse reaction velocities that were correlated to each other, we performed a global sensitivity analysis using the eFAST method, identifying the influential model parameters to fit to experimental data. Finally, we used published metabolomics data and performed PSO in order to find optimal parameter values. We selected the eight best-fit parameter sets based on the error between model predictions and experimental data. Importantly, the estimated parameter values were extremely consistent, with 25 of the 32 parameters varying less than 1\% across the eight best fits (Figure S2). Such consistency indicates the robustness of the parameter fitting technique and high confidence in the parameter values. Interestingly, the $V_{\max }$ value for glucokinase exhibits bimodality across the eight fitted parameter sets, showing multiple regimes that are equally capable of explaining the data.

The trained model was able to closely match the quantitative fold-change values measured experimentally for 17 metabolites used for parameter estimation (Figure 2). Furthermore, the 
model matched the fold-change data for time points that had been withheld as validation data, pointing to the ability to successfully predict data not used for model training. This further establishes the model's ability to match experimental data. We also compared model predictions to the qualitative direction of the change in metabolite levels upon stimulation with high glucose, based on literature review. These observed changes are indicated in the coloring of the nodes in Figure 1 and in Supplementary Figure S1.
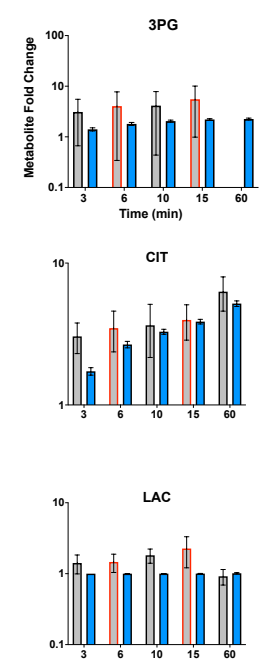
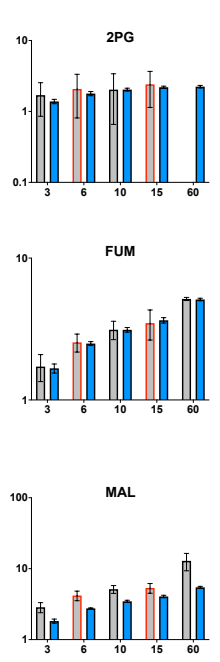
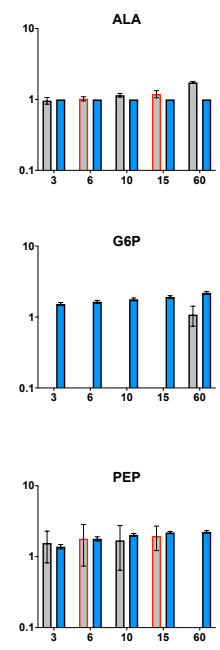
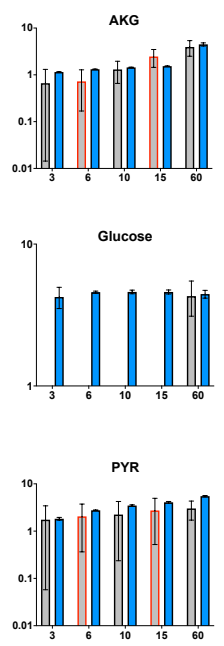
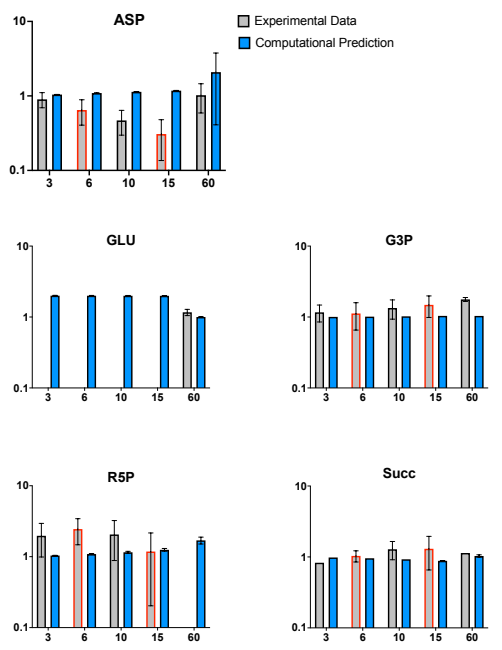

Figure 2: Model fit to experimental data. We trained the model to mass spectrometry data published by Spegel et al. and Malmgren et al., for the 3-, 10-, and 60-minute time points (gray bars with black outline) for 17 distinct metabolites. Model predictions (blue bars) match experimental measurements; the error bars represent the standard deviation of model predictions across the eight best-fit parameter sets. The experimental data for the 6and 15-minute time points (gray bars with red outline) were withheld as validation data to test the robustness of model predictions. Predicted fold-changes for metabolites found in both the cytosol and mitochondria are summed together as a total metabolite pool, in order to compare to the experimental data.

Besides the 17 unique metabolites used for model fitting, the model also predicts the foldchanges of 31 distinct metabolites. Most metabolites (88\%) qualitatively match the experimental fold-change direction, even metabolites that were not used in training. Therefore, the model provides a systems-level understanding of the effect of glucose stimulation. 


\subsection{Partial least squares regression modeling}

We developed a PLSR model correlating the reaction fluxes predicted by our calibrated model to reported insulin secretion. For each of the eight best-fit parameter sets, we predicted the flux through each reaction for the time period used in the experimental studies. We then performed PLSR analysis with the predicted fluxes as inputs and the measured fold-change in insulin secretion as outputs. Because each parameter set produced a distinct set of reaction fluxes, we generated and analyzed eight separate PLSR models. We found that PLSR models with three PLSR components best represented the data, capturing the majority of the variation in the outputs (Figure 3A).
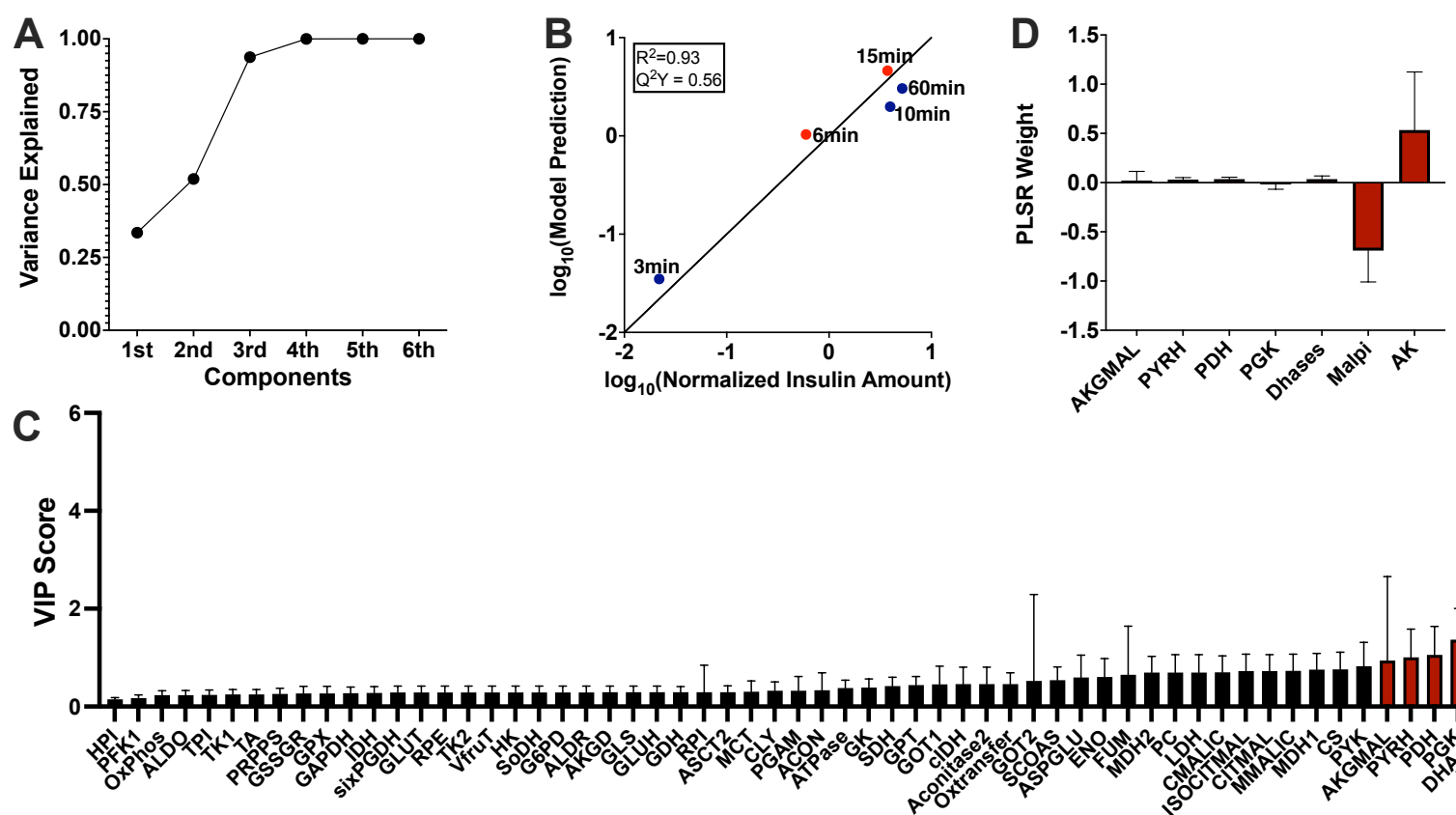

Figure 3: Partial least squares regression analysis. The PLSR model correlates the predicted flux through each reaction with measured insulin secretion amount at each time point of interest. Results shown are the average prediction across the PLSR model from each of the eight best-fit parameter sets. (A) Three PLSR components were used, as they collectively accounted for most of the variance. (B) Model predictions agreed with reported insulin amount, both for time points used for PLSR model building $(3,10$, and 60 minutes, blue circles), and for those held out as validation ( 6 and 15 minutes, orange circles). The PLSR models had an average $R^{2}$ value of 0.93 and $Q^{2} Y$ value of 0.56 . (C) We assessed the VIP scores for each metabolic reaction flux, shown in increasing order. Reactions with VIP scores greater than one are shown with red bars. (D) We analyzed the PLSR model weights associated with each reaction flux that were determined to be impactful. The weight shows 
how a change in flux value will affect insulin secretion. A positive weight indicates that increasing the flux value will increase insulin secretion.

The PLSR models agreed well with the experimental data (Figure 3B), both for the data used in training and data withheld for validation. The average $\mathrm{R}^{2}$ value across the models was 0.93 , and the $Q^{2} Y$ was 0.56 . We note that the low $Q^{2} Y$ value is likely due to the low number of time points used to develop the model, as the $Q^{2} Y$ performance metric assesses how the model would perform if trained on a subset of the available data and asked to predict the withheld data (leave-one-out cross validation). For a PLSR model with a small number of rows in the input matrix, leaving any data point out can substantially change the model's predictive power. Thus, the somewhat low $Q^{2} Y$ value is to be expected. Overall, this data-driven regression analysis approach reliably predicts the relationship between the flow of material through the network and the insulin produced.

We used the PLSR models to estimate the VIP scores, identifying the most important reaction fluxes that drive insulin secretion (Figure $\mathbf{3 C}$ ). The reactions with VIP scores greater than one are colored red, and the predictions are consistent across the eight models. Interestingly, several reaction fluxes with a VIP score above that threshold are either involved in the synthesis of energy (dhases and $a k$ ) or the shuttle of metabolites between cytosol and mitochondria (akgmal, malpi, and pyrh). The other two reactions predicted to be influential based on the VIP score were pyruvate dehydrogenase $(p d h)$ and phosphoglycerate kinase (pgk), involved in the conversion of pyruvate to acetyl-coA and 1,3-BPG to 3PG, respectively. The PLSR model weights for reactions with VIP scores above 1 (Figure 3D) provide information on whether a reaction is positively or negatively correlated to insulin secretion, and the strength of that correlation. The shuttling of malate between the cytosol and mitochondria (malpi) is negatively correlated in the model, suggesting that knocking down the reaction will drive an increase in insulin secretion. The $a k$ reaction has a predicted positive correlation with released insulin.

We also developed PLSR models at 1-minute time intervals for each short time course individually $(3,6,10$, and 15 -minutes). We again assessed the importance of each metabolic reaction on insulin produced per minute in a given time period. These results are shown in 
(Figure 4). Though most of the reactions with high VIP scores ( $a k, o x$, and malpi) are consistent between the total treatment time (Figure $3 \mathbf{C}$ ) and the short time courses, the glutamicoxaloacetic transaminase (got2) and fumarase (fum, alternatively called fumarate hydratase) reactions emerge as important in the short term. Overall, the kinetic and PLSR models allow us to predict targets for modulating intracellular metabolism and insulin secretion.

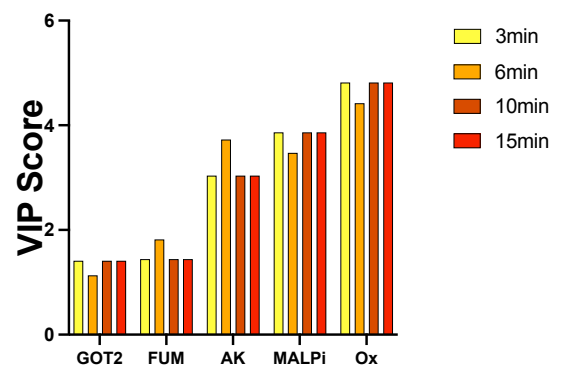

Figure 4: Short-term time course PLSR models. PLSR models were generated for each shorter time course of interest $(3,6,10$, and 15 minutes), correlating the average flux through each reaction with the insulin produced. The predicted VIP scores are shown for each time course.

\subsection{Effects of varying each model $V_{\max }$ value}

By combining the kinetic and PLSR models, we linked intracellular metabolism and insulin secretion. Using the integrated modeling framework, we predict how perturbing metabolic reactions affects insulin secretion and the whole metabolic network. We knocked down and increased the $V_{\max }$ value of each metabolic reaction by a factor of two and assessed the impact on each metabolite and on insulin secretion. The predicted fold-changes in the reaction fluxes compared to the baseline model with no perturbation are shown in Figure 5. We include the predicted change in insulin amount in the far-right column. 

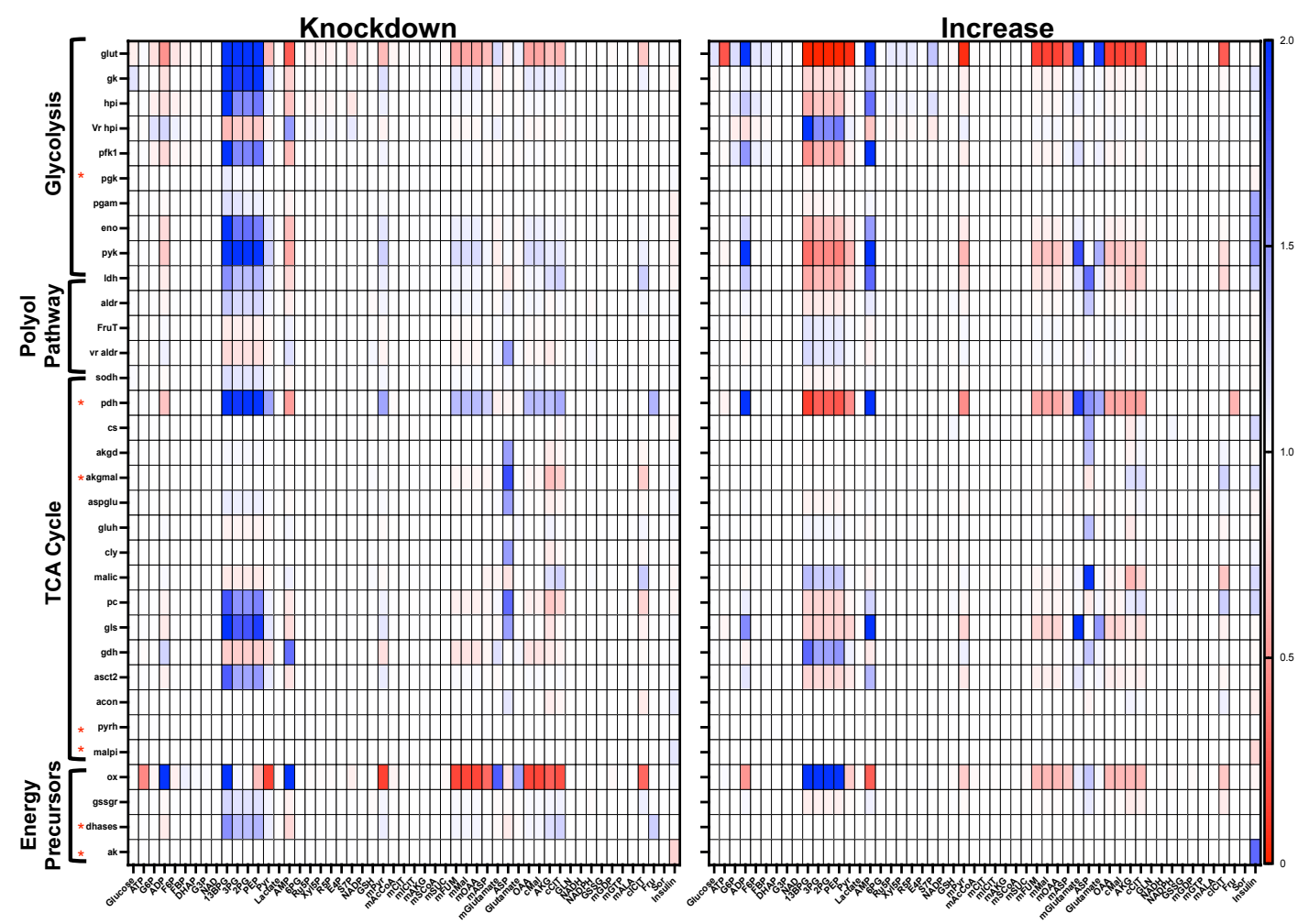

Figure 5: Effects of metabolic perturbations. We decreased (left) and increased (right) each reaction $V_{\max }$ value ( $y$-axis) by a factor of two and assessed the impact on all metabolites and insulin ( $x$-axis). The color bar indicates the effect of the perturbation relative to the base model with no perturbation.

As expected, increasing a $V_{\max }$ value led to opposite effect as decreasing the value for most reactions, but the amount by which those opposing perturbations affect cellular metabolism are not equal for every reaction velocity. For example, decreasing the glutaminase $(\mathrm{g} / \mathrm{s})$ reaction causes substantial increase in glycolytic intermediates, but increasing the rate does not lead to a comparable change in metabolite amounts. Similar trends can be seen with pyruvate kinase $(p y k)$, glucokinase $(g k)$, and glutaminase $(g / s)$, where the direction of the perturbation substantially and differentially impacts the metabolite levels in one direction more than the other. Perturbing some metabolic reactions is only impactful in one direction; for example, decreasing flux through the oxygen transfer reaction will lead to an increase in ATP, but increasing that reaction will have no impact on ATP levels. Similarly, increasing the activity of the $p d h$ or gls reactions will cause a significant increase in mitochondrial glutamate levels, but decreasing the $V_{\max }$ value of those enzymes is relatively ineffectual. 
Considering all of the metabolites in the model, we predict that downstream glycolytic metabolites (1,3-BPG, 2PG, 3PG, and PEP) are particularly susceptible to perturbations in the rest of the network, irrespective of the direction of that perturbation. Similarly, aspartate is sensitive to changes in the network, likely due to its ubiquity in the metabolic processes, both in the cytosol and mitochondria. In addition, perturbing the glut, $p d h, p d h, p y k$, and ox reactions elicits widespread changes in metabolite levels when perturbed, suggesting that the reactions are primary control points in the network that could cause a drastic shift in metabolism if targeted. Interestingly, perturbing glucose transporter (glut) or oxygen transfer (ox) decreases the levels of certain TCA cycle metabolites (mitochondrial fumarate, malate, oxaloacetate, and aspartate; cytosolic oxaloacetate, malate, $\alpha$-ketoglutarate, and citrate) whether the enzyme $V_{\max }$ value is increased or decreased. This indicates that some enzymes exert tight control over metabolic changes.

The regression analysis gives complementary information to the kinetic modeling, as it shows the metabolic reactions that are related to insulin. The VIP scores show which reaction fluxes correlate to insulin secretion, while the weights show whether those fluxes are positively or negatively correlated. We increased and decreased each metabolic reaction flux by a factor of two, and assessed the resulting predicted change in insulin secretion. These results are shown in the rightmost column of Figure 5. As indicated by the VIP scores and weights from the PLSR model (Figure 3), increasing the $a k$ and $a k g m a l$ reaction fluxes are predicted to increase insulin secretion. Increasing the pgk or malpi reaction fluxes leads to a decrease in insulin produced. Interestingly, reactions with lower VIP scores are also predicted to affect insulin secretion, including the pgam, eno, pyk, Idh, mitochondrial malic, and $p c$, since increasing the $V_{\max }$ value for these reactions increases insulin secretion.

\subsection{Effects of metformin on pancreatic $\beta$-cell metabolism}

Metformin was initially discovered as an antimalarial agent, but has become the leading diabetes drug in use because of its ability to lower blood glucose levels in the body[86]. 
Metformin primarily acts on the peripheral tissues and organs, reducing hepatic glucose production and increasing skeletal muscle glucose uptake. Though it is unclear how or if the drug affects pancreatic $\beta$-cells, one proposed hypothesis is that it reduces the cells' uptake of glucose, thereby acting as a protective mechanism to avoid overactivity and cellular exhaustion[69,71,72]. We tested the effects of metformin with our integrated modeling framework by decreasing the $V_{\max }$ value of the glut reaction by $80 \%$. We analyzed the impact on the predicted insulin amount and the metabolic network (i.e., metabolites and reaction fluxes).

The PLSR model ranked the flux through the glucose transporter as a relatively uninfluential reaction, with an average VIP score of 0.3 . Thus, it is not unexpected that decreasing the $V_{\max }$ of the glut reaction does not change the predicted insulin secretion. This is consistent with the field's consensus that the availability of glucose, and not its transport rate, is believed to be the driving factor modulating insulin secretion. This is because the glucose transporter has a high $K_{\mathrm{m}}$ value, which causes its observed "glucose sensing" ability[1,87].

We then assessed the impact of the simulated perturbation of the glut reaction on the kinetic model (Figure 6). As expected, we see a decrease in intracellular glucose levels. Similarly, upstream glycolytic metabolites are predicted to decrease, as do most PPP and TCA cycle intermediates. Flux through the reactions involving those metabolite levels is also substantially decreased, compared to the unperturbed system. Due to the simulated metformin perturbation, the fum, akgmal, and rpi reactions proceed in the opposite direction compared to the base model. Interestingly, the levels of downstream glycolytic metabolites (1,3-BPG, 3PG, 2PG, and PEP) are predicted to increase due to the simulated perturbation. This indicates that metformin leads to an accumulation or pooling of those metabolites. This accumulation is further confirmed by the prediction that flux through the glycolytic reactions involving these species (pgk, pgam, eno, and pyk) decreases. 


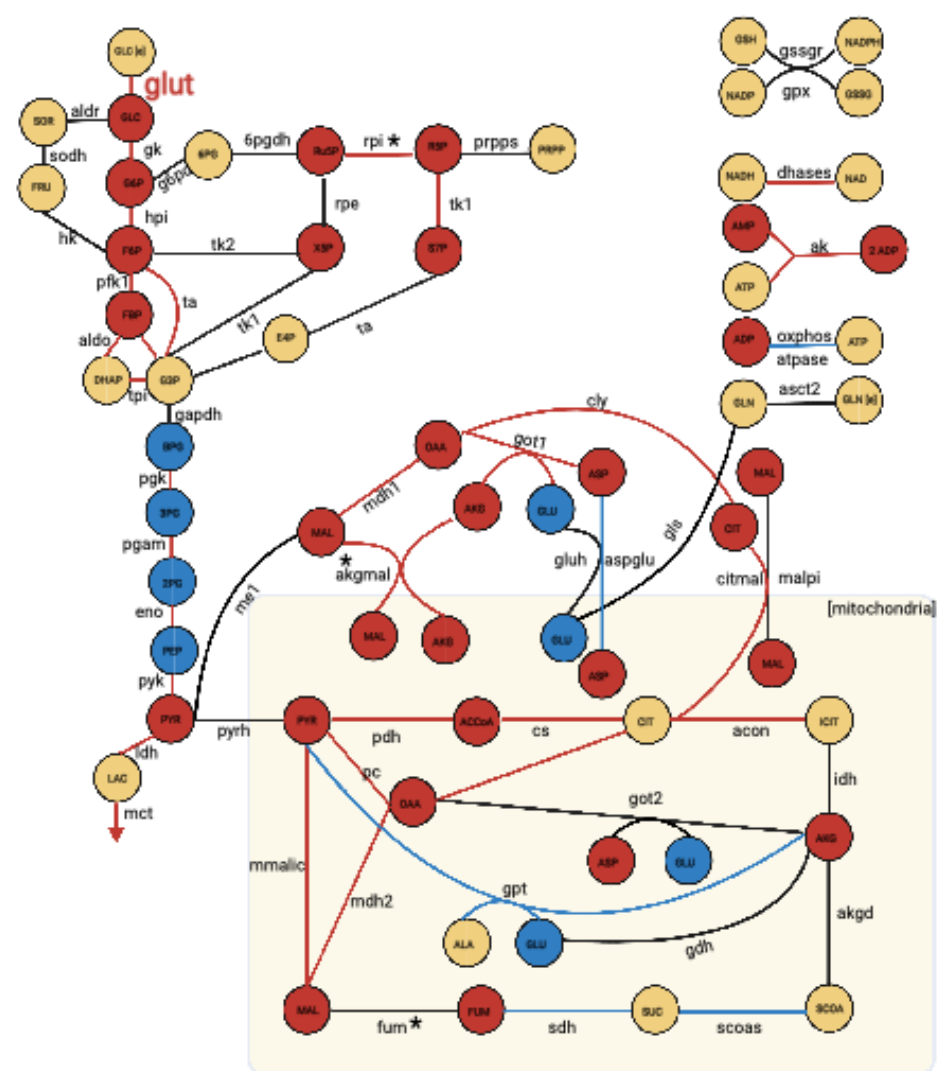

Increase in fold change Decrease in fold change No change in metabolite level

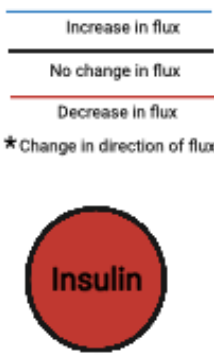

Figure 6: Effect of metformin treatment We implemented metformin as an $80 \%$ knockdown of the glucose transport (glut2) reaction and assessed the effect on the network, comparing metabolite levels, reaction fluxes, and insulin secretion to the unperturbed condition.

The simulated perturbation of glucose transport also affected some nucleotides, as we see a reduction in cellular ADP and AMP levels. However, ATP, NAD, NADH, NADP, and NADPH levels are mostly unchanged. The predicted decrease in ADP and AMP levels is driven by a decrease in the $a k, o x$, and dhases reactions, and an increase in the atpase reaction. Thus, the model predicts that the levels of the high-energy metabolite are robust to perturbations in glucose uptake.

\subsection{Predicted effects of agrimony on pancreatic $\beta$-cell metabolism}

Agrimony eupatoria (also called church steeples, in the Rosaceae family) is a traditional medicinal herb used to treat diabetes, as it has been shown to promote insulin secretion[75]. A common proposed mechanism by which agrimony induces increased insulin release is through 
antioxidant activities. It is well understood that diabetes and many other chronic illnesses are mediated through chronic inflammation, often driven by reactive oxygen or nitrogen species, which are affected by antioxidants[88-91]. It has been shown that the PPP serves to reduce inflammatory species, as the pathway drives the production of NADPH in the cell, which exerts a protective and anti-inflammatory influence on the $\beta$-cell[40-42,80]. To simulate the action of agrimony, we perturbed the glucose-6-phosphate dehydrogenase $(g 6 p d)$ reaction by increasing its flux, to simulate overexpression, as g6pd is the primary upstream controller of PPP activity.

The PLSR model predicted relatively minor increases in the insulin secretion of the cell (Figure 7), reported by a low VIP score. Though the metabolites and metabolic fluxes in the PPP are predicted to markedly increase compared to the baseline model condition, the perturbation caused relatively few other changes in the kinetic metabolic network: the model predicts no change in the metabolite levels or metabolic reaction fluxes in glycolysis, the TCA cycle, or the polyol pathway.

Additionally, the model predicts that increasing flux through the $g 6 p d$ reaction does not substantially affect the levels of energy precursors, ADP, ATP, NADP, NADH, and NAD. NADPH is the only energy precursor predicted to increase in response to increasing flux through the g6pd reaction. This indicates that effect of $g 6 p d$ perturbation is highly specific. Glutathione (GSH) levels in the cell are also predicted to be affected by the simulated targeting of $g 6 p d$, as NADPH is used to produced GSH in the glutathione reductase (gssgr) reaction. 


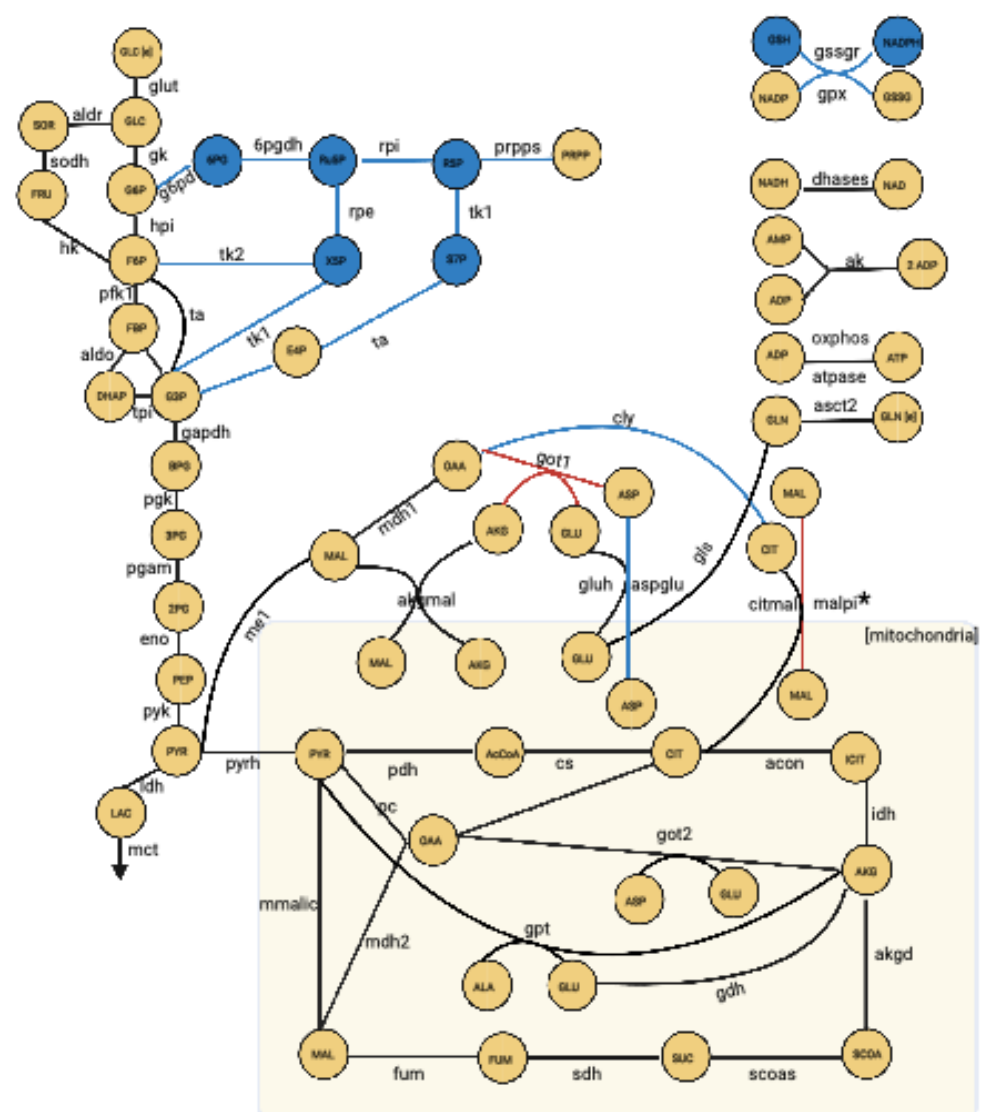

Increase in fold change Decrease in fold change No change in metabolite level

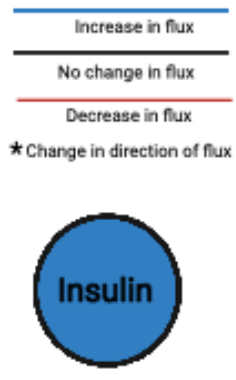

Figure 7: Effect of agrimony treatment. We implemented agrimony as a 5-fold increase in the g6pd reaction, and assessed the effect on the network, comparing metabolite levels, reaction fluxes, and insulin secretion to the unperturbed condition.

\subsection{Predicted effects of perturbing the $a k$ reaction}

The VIP scores and weights calculated in the PLSR analysis indicate the most impactful reaction fluxes involved in pancreatic $\beta$-cell metabolism and suggest the direction in which changing the associated $V_{\max }$ values would shift insulin secretion. Of the reactions with VIP scores greater than one, the ak reaction, which converts ATP and AMP into two molecules of ADP, was predicted to be the most impactful reaction positively correlated with insulin. The ak reaction is the primary mechanism by which cells maintain adenine nucleotide homeostasis, and it affects the AMP-activated protein kinase (AMPK) signaling cascade. The role of AMPK in insulin release is disputed, as it has been described both as a positive and a negative regulator of insulin secretion[92]. However, most published work affirms a link between ak and the ATP-mediated 
potassium channels, making the reaction of particular interest in $\beta$-cells. Thus, we upregulated the $V_{\max }$ value for the $a k$ reaction and assessed the effect on the entire metabolic network.

In our PLSR model, the ak reaction flux is strongly positively correlated to the secretion of insulin, as it has a high VIP score and positive weight. Our integrated modeling approach predicts that a five-fold increase in the $V_{\max }$ value for the ak reaction leads to a 1.7-fold increase in insulin secretion compared to the baseline model. However, increasing its $V_{\max }$ is not predicted to significantly affect metabolites in the kinetic network (Figure S3). This may identify $a k$ as a candidate treatment target for diabetes, as it could be used to increase insulin secretion, without disrupting the homeostasis of the $\beta$-cell.

\section{Discussion}

\subsection{Utility of our predictive modeling framework}

Computational modeling is a tool by which we can synthesize disparate information and data to generate novel predictions. In particular, modeling allows us to take a systems-level view of how individual parts of a metabolic network (i.e., reactions and metabolites) work together to generate observed behavior. Here, we have developed a predictive kinetic model that is able to capture the dynamics of metabolism in pancreatic $\beta$-cells. The model consists of glycolysis, glutaminolysis, the PPP, the TCA cycle, and polyol pathway, building upon previously published modeling efforts. The model has been trained to and validated with published qualitative and quantitative metabolomics data from the INS1 832/13 cell line collected in vitro. The calibrated kinetic model predicts metabolite concentrations and reaction fluxes. It is important to note that the computational model can differentiate between the levels of metabolites that are found in both the cytosol and mitochondria, whereas the mass spectrometry pipeline pools them together and cannot easily discriminate between metabolites in different cellular subcompartments. This is a further benefit of the kinetic modeling approach, as it can investigate the proportion of a metabolite pool in a particular compartment. 
We paired the kinetic model with regression analysis. Integrating a kinetic model with a PLSR model allows us to further analyze systems-level dynamics of the central carbon metabolic network in $\beta$-cells and relate the predicted metabolite levels and reaction fluxes to a cellularlevel response (insulin secretion). Though pairing kinetic modeling with data-driven modeling is a somewhat underutilized approach, it can be used to extend the predictive capabilities of kinetic modeling, thereby gaining novel insights.

The influential reactions predicted by combining kinetic and PLSR models agree with experimental observations. Our approach predicted that reactions involved in energy synthesis and transport between the cytosol and mitochondria strongly contribute to insulin secretion. Both of those cellular processes have been previously implicated with insulin production[9395]. We also predict the importance of the pyruvate dehydrogenase ( $p d h)$ enzyme. This enzyme has a key regulatory role in GSIS, as it controls the generation of ATP and other metabolic coupling factors that are directly responsible for the release of insulin[96,97]. This is because $p d h$ carries out the oxidative decarboxylation that converts pyruvate to acetyl-CoA, linking glycolysis to the TCA cycle. Thus, the $p d h$ reaction initiates mitochondrial metabolic processes (especially pyruvate cycling), which drive insulin secretion. Furthermore, decreasing $p d h$ has been shown to impair insulin secretion[98-100].

The ultimate goal of our modeling work is to identify novel mechanisms to treat diabetes. As a step towards this goal, we perturbed each model reaction and explored in detail the effect of pharmacologic interventions, both on metabolite levels and fluxes and on insulin secretion. We thus used the model for hypothesis generation and testing. Additionally, our modeling framework can identify the time-dependent nature of insulin secretion, as shown by comparing the overall PLSR model (generated with all five time points) with the short-term PLSR models generated. We predict that the got2 and fum reactions are important only in the short time course. Mitochondrial glutamine and glutamate metabolism, in part mediated by got2, is a primary driver of incretin-induced insulin secretion (IIIS) and is known to metabolically connect pancreatic alpha cells and $\beta$-cells[101,102]. Fumarase has been linked to insulin secretory 
activity, as deletion of the enzyme led to a decrease in GSIS[103]. It is notable that those two metabolic reactions emerge as impactful, as this highlights the time-dependent and dynamic nature of $\beta$-cell activity and suggests it may be worthwhile to investigate the impact of perturbing the got2 and fum reactions in vivo.

Excitingly, our model predictions of the effects of metabolic perturbations induced by pharmacologic agents agree with literature evidence. Lamontagne et al. treated INS1 cells with metformin at varying extracellular glucose levels[69]. They showed that metformin caused a decrease in GSIS at intermediate glucose conditions, which supports the PLSR model's prediction, as the glucose transporter has a negative, albeit small, weight, indicating a negative correlation with insulin. Lamontagne and coworkers also measured metabolite levels following metformin treatment, proposing that the treatment increased cellular glutamate levels, did not affect the concentrations of metabolites such as G3P, GSH, GSSG, NAD, or NADH, and attenuated the effect of high glucose levels on TCA cycle metabolites. Each of those experimental measurements is also seen in our model predictions, suggesting the implemented mechanism (reducing glucose transport) is a promising hypothesis.

Our model unexpectedly predicted that altering the $g 6 p d$ reaction (simulating agrimony supplementation) led to an increase in glutathione levels in the cell. Glutathione is among the most well-studied natural antioxidants, capable of preventing cell damage incurred by reactive oxygen and nitrogen species[104-107]. This emergent and unanticipated prediction from our model supports the potential utility of agrimony supplementation among diabetes patients. It is also interesting that the model was able to provide confirmation to a hypothesis regarding the mechanism of action of agrimony; namely, we predict that agrimony targets the $g 6 p d$ reaction in the PPP. Increased flux through this reaction can cause increased levels of antioxidants, which reduce cellular inflammation and enable the $\beta$-cell to properly function[31,108-111].

The $a k$ reaction is predicted to increase insulin secretion without substantially changing the metabolic network dynamics. This may be due to its involvement in the AMP-activated protein 
kinase signaling pathway, which was not included in our modeling effort but has been shown to influence insulin secretion. Targeting the $a k$ reaction may be of particular clinical interest, as it can increase insulin production and reduce the hyperglycemic pressures experienced by patients without affecting the survival of the $\beta$-cell. An experimental drug, bis(adenosine)-5'pentaphosphate, which targets the $a$ k reaction, has previously been studied as a vasoconstrictor[112]. Our work suggests that it may be of use in diabetes, repurposed to increase insulin secretion. Future work can assess its viability as an anti-diabetic treatment strategy.

\subsection{Study Limitations}

Though the model captures the dynamics of pancreatic $\beta$-cell metabolism and can be applied to study clinically relevant interventions, there are some limitations that affect the utility and applicability of the computational model. The model does not account for heterogeneity within a population of cells and does not consider the metabolic interactions between $\beta$-cell and the other islet cells, such as $\alpha$ - or $\delta$-cells. The model is built based upon prior modeling efforts from $\beta$-cells and other cell types. We used experimental data from the INS1 832/13 cell line to make the reaction velocities specific to the pancreatic $\beta$-cell; however, the form of the rate equations can also be refined based on $\beta$-cell-specific data as they become available. We focused on central carbon metabolism, but there are additional pathways that could be included. For example, the degradation of free fatty acids is thought to impair insulin secretion in $\beta$-cell, and may be of particular interest in diabetes; this is an avenue for future research. More broadly, future iterations of this work may address these limitations.

\section{Conclusions}

We present a novel kinetic model that can effectively be used to study the dynamics of central carbon metabolism in pancreatic $\beta$-cells. The model goes beyond existing models and consists of key pathways and metabolites known to be important in GSIS. The model has been trained and validated with published data from the INS1 cell line. The model simulates the effects of 
metabolic perturbations, predicting the metabolite levels and flux distributions upon knockdown or upregulation of specific enzymatic reactions. We pair the kinetic model with a data-driven modeling approach, thereby linking intracellular metabolism to insulin secretion. The model is a promising step towards effectively using in silico techniques to generate novel insights into pancreatic $\beta$-cells. Thus, our work complements experimental studies and can be used to identify novel treatment strategies for diabetes.

\section{Acknowledgments}

The authors thank members of the Finley research group and the Pancreatic Beta Cell Consortium (especially the Metabolomics sub-group) for helpful discussions. Computation for the work described in this paper was supported by the University of Southern California's Center for High-Performance Computing (https://hpcc.usc.edu).

\section{Author Contributions}

SDF conceived of and designed the research. PG constructed the model and performed the simulations and analyses. DZ, SEF, KW, and NAG contributed to the interpretation of the results. All authors contributed to writing the manuscript and approved of its final version. 
Computational modeling of pancreatic $\beta$-cell metabolism

\section{References}

1. MacDonald PE, Joseph JW, Rorsman P. Glucose-sensing mechanisms in pancreatic $\beta$-cells. Philos Trans R Soc B Biol Sci. 2005 Dec 29;360(1464):2211-25.

2. Schuit FC, Huypens P, Heimberg H, Pipeleers DG. Glucose Sensing in Pancreatic $\beta$-Cells: A Model for the Study of Other Glucose-Regulated Cells in Gut, Pancreas, and Hypothalamus. Diabetes. 2001 Jan 1;50(1):1-11.

3. Detimary P, Van den Berghe G, Henquin J-C. Concentration Dependence and Time Course of the Effects of Glucose on Adenine and Guanine Nucleotides in Mouse Pancreatic Islets. J Biol Chem. 1996 Aug;271(34):20559-65.

4. Rutter GA, Georgiadou E, Martinez-Sanchez A, Pullen TJ. Metabolic and functional specialisations of the pancreatic beta cell: gene disallowance, mitochondrial metabolism and intercellular connectivity. Diabetologia. 2020 Oct 1;63(10):1990-8.

5. Meglasson MD, Matschinsky FM. New perspectives on pancreatic islet glucokinase. Am J Physiol-Endocrinol Metab. 1984 Jan 1;246(1):E1-13.

6. Spégel P, Mulder H. Metabolomics Analysis of Nutrient Metabolism in $\beta$-Cells. J Mol Biol. 2020 Mar 6;432(5):1429-45.

7. Aronoff SL, Berkowitz K, Shreiner B, Want L. Glucose Metabolism and Regulation: Beyond Insulin and Glucagon. Diabetes Spectr. 2004 Jul 1;17(3):183-90.

8. Nicholls DG. The Pancreatic $\beta$-Cell: A Bioenergetic Perspective. Physiol Rev. 2016 Oct 1;96(4):1385-447.

9. Mulder H. Metabolic coupling in pancreatic beta cells: lipolysis revisited. Diabetologia. 2016 Dec 1;59(12):2510-3.

10. Wortham $M$, Sander M. Mechanisms of $\beta$-cell functional adaptation to changes in workload. Diabetes Obes Metab. 2016 Sep;18(Suppl 1):78-86.

11. Haythorne E, Rohm M, van de Bunt M, Brereton MF, Tarasov AI, Blacker TS, et al. Diabetes causes marked inhibition of mitochondrial metabolism in pancreatic $\beta$-cells. Nat Commun. 2019 Jun 6;10(1):2474.

12. Clark A, Jones LC, de Koning E, Hansen BC, Matthews DR. Decreased insulin secretion in type 2 diabetes: a problem of cellular mass or function? Diabetes. 2001 Feb;50 Suppl 1:S169-171.

13. Holman RR, Clark A, Rorsman P. $\beta$-cell secretory dysfunction: a key cause of type 2 diabetes. Lancet Diabetes Endocrinol. 2020 May 1;8(5):370. 
14. Volkova S, Matos MRA, Mattanovich M, Marín de Mas I. Metabolic Modelling as a Framework for Metabolomics Data Integration and Analysis. Metabolites. 2020 Jul 24;10(8):E303.

15. Topp B, Promislow K, Devries G, Miura RM, Finegood DT. A Model of $\beta$-Cell Mass, Insulin, and Glucose Kinetics: Pathways to Diabetes. J Theor Biol. 2000 Oct 21;206(4):605-19.

16. Bertram R, Gram Pedersen M, Luciani DS, Sherman A. A simplified model for mitochondrial ATP production. J Theor Biol. 2006 Dec 21;243(4):575-86.

17. Magnus G, Keizer J. Minimal model of beta-cell mitochondrial Ca2+ handling. Am J Physiol. 1997 Aug;273(2 Pt 1):C717-733.

18. Magnus G, Keizer J. Model of beta-cell mitochondrial calcium handling and electrical activity. I. Cytoplasmic variables. Am J Physiol. 1998 Apr;274(4):C1158-1173.

19. Yugi K, Tomita M. A general computational model of mitochondrial metabolism in a whole organelle scale. Bioinforma Oxf Engl. 2004 Jul 22;20(11):1795-6.

20. Fridlyand LE, Philipson LH. Glucose sensing in the pancreatic beta cell: a computational systems analysis. Theor Biol Med Model. 2010 May 24;7:15.

21. Jiang N, Cox RD, Hancock JM. A kinetic core model of the glucose-stimulated insulin secretion network of pancreatic $\beta$ cells. Mamm Genome. 2007 Jul 1;18(6):508-20.

22. Roy M, Finley SD. Computational Model Predicts the Effects of Targeting Cellular Metabolism in Pancreatic Cancer. Front Physiol [Internet]. 2017 [cited 2021 Aug 9];0. Available from: https://www.frontiersin.org/articles/10.3389/fphys.2017.00217/full

23. Cortassa S, Caceres V, Bell LN, O’Rourke B, Paolocci N, Aon MA. From Metabolomics to Fluxomics: A Computational Procedure to Translate Metabolite Profiles into Metabolic Fluxes. Biophys J. 2015 Jan 6;108(1):163-72.

24. Cornish-Bowden A. One hundred years of Michaelis-Menten kinetics. Perspect Sci. 2015 Mar 1;4:3-9.

25. Ulusu NN. Evolution of Enzyme Kinetic Mechanisms. J Mol Evol. 2015;80(5-6):251-7.

26. Curve Fitting Toolbox [Internet]. [cited 2021 Aug 9]. Available from: https://www.mathworks.com/products/curvefitting.html

27. German MS. Glucose sensing in pancreatic islet beta cells: the key role of glucokinase and the glycolytic intermediates. Proc Natl Acad Sci. 1993 Mar 1;90(5):1781-5.

28. Efrat S, Tal M, Lodish HF. The pancreatic beta-cell glucose sensor. Trends Biochem Sci. 1994 Dec;19(12):535-8. 
29. Matschinsky FM, Wilson DF. The Central Role of Glucokinase in Glucose Homeostasis: A Perspective 50 Years After Demonstrating the Presence of the Enzyme in Islets of Langerhans. Front Physiol [Internet]. 2019 [cited 2021 Aug 9];0. Available from: https://www.frontiersin.org/articles/10.3389/fphys.2019.00148/full

30. Brun T, Maechler P. Beta-cell mitochondrial carriers and the diabetogenic stress response. Biochim Biophys Acta BBA - Mol Cell Res. 2016 Oct 1;1863(10):2540-9.

31. Ge T, Yang J, Zhou S, Wang Y, Li Y, Tong X. The Role of the Pentose Phosphate Pathway in Diabetes and Cancer. Front Endocrinol. 2020 Jun 9;11:365.

32. Kalwat MA, Cobb MH. Mechanisms of the Amplifying Pathway of Insulin Secretion in the $\beta$ Cell. Pharmacol Ther. 2017 Nov;179:17-30.

33. Huang $\mathrm{M}$, Joseph JW. Metabolomic analysis of pancreatic $\beta$-cell insulin release in response to glucose. Islets. 2012 May 1;4(3):210-22.

34. Lorenz MA, El Azzouny MA, Kennedy RT, Burant CF. Metabolome response to glucose in the $\beta$-cell line INS-1 832/13. J Biol Chem. 2013 Apr 12;288(15):10923-35.

35. Goehring I, Sauter NS, Catchpole G, Assmann A, Shu L, Zien KS, et al. Identification of an intracellular metabolic signature impairing beta cell function in the rat beta cell line INS$1 \mathrm{E}$ and human islets. Diabetologia. $2011 \mathrm{Jul}$ 28;54(10):2584.

36. Jitrapakdee S, Wutthisathapornchai A, Wallace JC, MacDonald MJ. Regulation of insulin secretion: role of mitochondrial signalling. Diabetologia. 2010 Jun;53(6):1019-32.

37. Newgard CB, McGarry JD. Metabolic coupling factors in pancreatic beta-cell signal transduction. Annu Rev Biochem. 1995;64:689-719.

38. Newgard CB, Lu D, Jensen MV, Schissler J, Boucher A, Burgess S, et al. Stimulus/Secretion Coupling Factors in Glucose-Stimulated Insulin Secretion: Insights Gained From a Multidisciplinary Approach. Diabetes. 2002 Dec 1;51(suppl 3):S389-93.

39. Prentki M, Matschinsky FM, Madiraju SRM. Metabolic Signaling in Fuel-Induced Insulin Secretion. Cell Metab. 2013 Aug 6;18(2):162-85.

40. Gray JP, Alavian KN, Jonas EA, Heart EA. NAD kinase regulates the size of the NADPH pool and insulin secretion in pancreatic $\beta$-cells. Am J Physiol-Endocrinol Metab. $2012 \mathrm{Jul}$ 15;303(2):E191-9.

41. Luo X, Li R, Yan L-J. Roles of Pyruvate, NADH, and Mitochondrial Complex I in Redox Balance and Imbalance in $\beta$ Cell Function and Dysfunction. J Diabetes Res. 2015 Oct 19;2015:e512618. 
42. Xiao W, Wang R-S, Handy DE, Loscalzo J. NAD(H) and NADP(H) Redox Couples and Cellular Energy Metabolism. Antioxid Redox Signal. 2018 Jan 20;28(3):251-72.

43. Nishimura-Yabe C. [Aldose reductase in the polyol pathway: a potential target for the therapeutic intervention of diabetic complications]. Nihon Yakurigaku Zasshi Folia Pharmacol Jpn. 1998 Mar 1;111(3):137-45.

44. Gabbay KH, Tze WJ. Inhibition of Glucose-Induced Release of Insulin by Aldose Reductase Inhibitors. Proc Natl Acad Sci. 1972 Jun 1;69(6):1435-9.

45. Robertson RP. Chronic Oxidative Stress as a Central Mechanism for Glucose Toxicity in Pancreatic Islet Beta Cells in Diabetes *. J Biol Chem. 2004 Oct 8;279(41):42351-4.

46. Tang W, Martin KA, Hwa J. Aldose Reductase, Oxidative Stress, and Diabetic Mellitus. Front Pharmacol [Internet]. 2012 [cited 2021 Aug 9];0. Available from: https://www.frontiersin.org/articles/10.3389/fphar.2012.00087/full

47. McKay MD, Beckman RJ, Conover WJ. A Comparison of Three Methods for Selecting Values of Input Variables in the Analysis of Output from a Computer Code. Technometrics. 1979;21(2):239-45.

48. Nazaret C, Heiske M, Thurley K, Mazat J-P. Mitochondrial energetic metabolism: A simplified model of TCA cycle with ATP production. J Theor Biol. 2009 Jun 7;258(3):45564.

49. Nielsen K, Sørensen PG, Hynne F, Busse H-G. Sustained oscillations in glycolysis: an experimental and theoretical study of chaotic and complex periodic behavior and of quenching of simple oscillations. Biophys Chem. 1998 May 5;72(1):49-62.

50. WebPlotDigitizer - Extract data from plots, images, and maps [Internet]. [cited 2021 Aug 9]. Available from: https://automeris.io/WebPlotDigitizer/citation.html

51. Spégel P, Sharoyko VV, Goehring I, Danielsson APH, Malmgren S, Nagorny CLF, et al. Time-resolved metabolomics analysis of $\beta$-cells implicates the pentose phosphate pathway in the control of insulin release. Biochem J. 2013 Mar 15;450(3):595-605.

52. Malmgren S, Spégel P, Danielsson APH, Nagorny CL, Andersson LE, Nitert MD, et al. Coordinate Changes in Histone Modifications, mRNA Levels, and Metabolite Profiles in Clonal INS-1 832/13 $\beta$-Cells Accompany Functional Adaptations to Lipotoxicity *. J Biol Chem. 2013 Apr 26;288(17):11973-87.

53. Spégel P, Malmgren S, Sharoyko VV, Newsholme P, Koeck T, Mulder H. Metabolomic analyses reveal profound differences in glycolytic and tricarboxylic acid cycle metabolism in glucose-responsive and -unresponsive clonal $\beta$-cell lines. Biochem J. 2011 Apr 1;435(1):277-84. 
54. Spégel P, Andersson LE, Storm P, Sharoyko V, Göhring I, Rosengren AH, et al. Unique and Shared Metabolic Regulation in Clonal $\beta$-Cells and Primary Islets Derived From Rat Revealed by Metabolomics Analysis. Endocrinology. 2015 Jun 1;156(6):1995-2005.

55. Huang M, Joseph JW. Assessment of the Metabolic Pathways Associated With GlucoseStimulated Biphasic Insulin Secretion. Endocrinology. 2014 May 1;155(5):1653-66.

56. El-Azzouny M, Evans CR, Treutelaar MK, Kennedy RT, Burant CF. Increased glucose metabolism and glycerolipid formation by fatty acids and GPR40 receptor signaling underlies the fatty acid potentiation of insulin secretion. J Biol Chem. 2014 May 9;289(19):13575-88.

57. Guay C, Joly E, Pepin E, Barbeau A, Hentsch L, Pineda M, et al. A role for cytosolic isocitrate dehydrogenase as a negative regulator of glucose signaling for insulin secretion in pancreatic ß-cells. PloS One. 2013;8(10):e77097.

58. Mugabo Y, Zhao S, Lamontagne J, Al-Mass A, Peyot M-L, Corkey BE, et al. Metabolic fate of glucose and candidate signaling and excess-fuel detoxification pathways in pancreatic $\beta$-cells. J Biol Chem. 2017 May 5;292(18):7407-22.

59. Andersson LE, Shcherbina L, Al-Majdoub M, Vishnu N, Arroyo CB, Carrara JA, et al. Glutamine-Elicited Secretion of Glucagon-Like Peptide 1 Is Governed by an Activated Glutamate Dehydrogenase. Diabetes. 2018 Mar 1;67(3):372-84.

60. Stamenkovic JA, Andersson LE, Adriaenssens AE, Bagge A, Sharoyko VV, Gribble F, et al. Inhibition of the malate-aspartate shuttle in mouse pancreatic islets abolishes glucagon secretion without affecting insulin secretion. Biochem J. 2015 May 15;468(1):49-63.

61. Saltelli A, Tarantola S, Chan KP-S. A Quantitative Model-Independent Method for Global Sensitivity Analysis of Model Output. Technometrics. 1999 Feb 1;41(1):39-56.

62. Kennedy J, Eberhart R. Particle swarm optimization. In: Proceedings of ICNN'95 International Conference on Neural Networks. 1995. p. 1942-8 vol.4.

63. Stapor P, Weindl D, Ballnus B, Hug S, Loos C, Fiedler A, et al. PESTO: Parameter EStimation TOolbox. Bioinformatics. 2018 Feb 15;34(4):705-7.

64. Helland IS. Some theoretical aspects of partial least squares regression. Chemom Intell Lab Syst. 2001 Oct 28;58(2):97-107.

65. de Jong S. SIMPLS: An alternative approach to partial least squares regression. Chemom Intell Lab Syst. 1993 Mar 1;18(3):251-63.

66. Akter S, D'Ambra J, Ray P. An evaluation of PLS based complex models: the roles of power analysis, predictive relevance and GoF index. 2011;9. 
67. Akarachantachote N, Chadcham S, Saithanu K. CUTOFF THRESHOLD OF VARIABLE IMPORTANCE IN PROJECTION FOR VARIABLE SELECTION. Int J Pure Apllied Math [Internet]. 2014 Jul 17 [cited 2021 Aug 9];94(3). Available from: http://www.ijpam.eu/contents/2014-94-3/2/

68. Rena G, Hardie DG, Pearson ER. The mechanisms of action of metformin. Diabetologia. 2017;60(9):1577-85.

69. Lamontagne J, Al-Mass A, Nolan CJ, Corkey BE, Madiraju SRM, Joly E, et al. Identification of the signals for glucose-induced insulin secretion in INS1 (832/13) $\beta$-cells using metformin-induced metabolic deceleration as a model. J Biol Chem. $2017 \mathrm{Nov}$ 24;292(47):19458-68.

70. Tajima K, Shirakawa J, Okuyama T, Kyohara M, Yamazaki S, Togashi Y, et al. Effects of metformin on compensatory pancreatic $\beta$-cell hyperplasia in mice fed a high-fat diet. Am J Physiol-Endocrinol Metab. 2017 Sep 1;313(3):E367-80.

71. Gelin L, Li J, Corbin KL, Jahan I, Nunemaker CS. Metformin Inhibits Mouse Islet Insulin Secretion and Alters Intracellular Calcium in a Concentration-Dependent and DurationDependent Manner near the Circulating Range. J Diabetes Res. 2018 Mar 18;2018:e9163052.

72. Metformin Preserves $\beta$-Cell Compensation in Insulin Secretion and Mass Expansion in Prediabetic Nile Rats [Internet]. [cited 2021 Aug 2]. Available from: https://www.ncbi.nlm.nih.gov/pmc/articles/PMC7794750/

73. Li WL, Zheng HC, Bukuru J, De Kimpe N. Natural medicines used in the traditional Chinese medical system for therapy of diabetes mellitus. J Ethnopharmacol. 2004 May 1;92(1):121.

74. Traditional plant treatments for diabetes mellitus: pharmaceutical foods | British Journal of Nutrition | Cambridge Core [Internet]. [cited 2021 Aug 9]. Available from: https://www.cambridge.org/core/journals/british-journal-ofnutrition/article/traditional-plant-treatments-for-diabetes-mellitus-pharmaceuticalfoods/4669ADA39C5AFD59983238857A772C69

75. Gray AM, Flatt PR. Actions of the traditional anti-diabetic plant, Agrimony eupatoria (agrimony): effects on hyperglycaemia, cellular glucose metabolism and insulin secretion. Br J Nutr. 1998 Jul;80(1):109-14.

76. Santos TN, Costa G, Ferreira JP, Liberal J, Francisco V, Paranhos A, et al. Antioxidant, AntiInflammatory, and Analgesic Activities of Agrimonia eupatoria L. Infusion. Evid-Based Complement Altern Med ECAM. 2017;2017:8309894. 
77. Sakai K, Matsumoto K, Nishikawa T, Suefuji M, Nakamaru K, Hirashima Y, et al. Mitochondrial reactive oxygen species reduce insulin secretion by pancreatic $\beta$-cells. Biochem Biophys Res Commun. 2003 Jan 3;300(1):216-22.

78. Tiganis T. Reactive oxygen species and insulin resistance: the good, the bad and the ugly. Trends Pharmacol Sci. 2011 Feb 1;32(2):82-9.

79. Matsuoka T, Kajimoto Y, Watada H, Kaneto H, Kishimoto M, Umayahara Y, et al. Glycation-dependent, reactive oxygen species-mediated suppression of the insulin gene promoter activity in HIT cells. J Clin Invest. 1997 Jan 1;99(1):144-50.

80. McCarty MF, Barroso-Aranda J, Contreras F. NADPH oxidase mediates glucolipotoxicityinduced beta cell dysfunction--clinical implications. Med Hypotheses. 2010 Mar;74(3):596-600.

81. Glucose Toxicity in $\beta$-Cells: Type 2 Diabetes, Good Radicals Gone Bad, and the Glutathione Connection | Diabetes [Internet]. [cited 2021 Aug 9]. Available from: https://diabetes.diabetesjournals.org/content/52/3/581

82. Newsholme P, Keane KN, Carlessi R, Cruzat V. Oxidative stress pathways in pancreatic $\beta$ cells and insulin-sensitive cells and tissues: importance to cell metabolism, function, and dysfunction. Am J Physiol-Cell Physiol. 2019 Sep 1;317(3):C420-33.

83. Tiwari M. Glucose 6 phosphatase dehydrogenase (G6PD) and neurodegenerative disorders: Mapping diagnostic and therapeutic opportunities. Genes Dis. 2017 Sep 23;4(4):196-203.

84. Yang H-C, Stern A, Chiu DT-Y. G6PD: A hub for metabolic reprogramming and redox signaling in cancer. Biomed J. 2021 Jun 1;44(3):285-92.

85. G6PD protects from oxidative damage and improves healthspan in mice / Nature Communications [Internet]. [cited 2021 Aug 9]. Available from: https://www.nature.com/articles/ncomms10894

86. Bailey CJ. Metformin: historical overview. Diabetologia. 2017 Sep;60(9):1566-76.

87. Thorens B. GLUT2, glucose sensing and glucose homeostasis. Diabetologia. 2015 Feb $1 ; 58(2): 221-32$.

88. Wellen KE, Hotamisligil GS. Inflammation, stress, and diabetes. J Clin Invest. 2005 May 2;115(5):1111-9.

89. Tsalamandris S, Antonopoulos AS, Oikonomou E, Papamikroulis G-A, Vogiatzi G, Papaioannou S, et al. The Role of Inflammation in Diabetes: Current Concepts and Future Perspectives. Eur Cardiol Rev. 2019 Apr;14(1):50-9. 
90. Bajaj S, Khan A. Antioxidants and diabetes. Indian J Endocrinol Metab. 2012 Dec;16(Suppl 2):S267-71.

91. Kaneto H, Kajimoto Y, Miyagawa J, Matsuoka T, Fujitani Y, Umayahara Y, et al. Beneficial effects of antioxidants in diabetes: possible protection of pancreatic beta-cells against glucose toxicity. Diabetes. 1999 Dec;48(12):2398-406.

92. Fu A, Eberhard CE, Screaton RA. Role of AMPK in pancreatic beta cell function. Mol Cell Endocrinol. 2013 Feb 25;366(2):127-34.

93. Fu Z, Gilbert ER, Liu D. Regulation of Insulin Synthesis and Secretion and Pancreatic BetaCell Dysfunction in Diabetes. Curr Diabetes Rev. 2013 Jan 1;9(1):25-53.

94. Regulation of ATP/ADP in Pancreatic Islets | Diabetes [Internet]. [cited 2021 Aug 9]. Available from: https://diabetes.diabetesjournals.org/content/53/2/401

95. Joseph JW, Jensen MV, Ilkayeva O, Palmieri F, Alárcon C, Rhodes CJ, et al. The mitochondrial citrate/isocitrate carrier plays a regulatory role in glucose-stimulated insulin secretion. J Biol Chem. 2006 Nov 24;281(47):35624-32.

96. Srinivasan M, Choi CS, Ghoshal P, Pliss L, Pandya JD, Hill D, et al. $\beta$-Cell-specific pyruvate dehydrogenase deficiency impairs glucose-stimulated insulin secretion. Am J Physiol Endocrinol Metab. 2010 Dec;299(6):E910-7.

97. Sugden MC, Bulmer K, Augustine D, Holness MJ. Selective Modification of Pyruvate Dehydrogenase Kinase Isoform Expression in Rat Pancreatic Islets Elicited by Starvation and Activation of Peroxisome Proliferator-Activated Receptor- $\alpha$ : Implications for Glucose-Stimulated Insulin Secretion. Diabetes. 2001 Dec 1;50(12):2729-36.

98. Wang X, Lai S, Ye Y, Hu Y, Pan D, Bai X, et al. Conditional knockout of pyruvate dehydrogenase in mouse pancreatic $\beta$-cells causes morphological and functional changes. Mol Med Rep. 2020 Apr;21(4):1717-26.

99. Krus U, Kotova O, Spégel P, Hallgard E, Sharoyko VV, Vedin A, et al. Pyruvate dehydrogenase kinase 1 controls mitochondrial metabolism and insulin secretion in INS1 832/13 clonal beta-cells. Biochem J. 2010 Jul 1;429(1):205-13.

100. Zhang S, Hulver MW, McMillan RP, Cline MA, Gilbert ER. The pivotal role of pyruvate dehydrogenase kinases in metabolic flexibility. Nutr Metab. 2014 Feb 12;11(1):10.

101. Yokoi N, Gheni G, Takahashi H, Seino S. $\beta$-Cell glutamate signaling: Its role in incretininduced insulin secretion. J Diabetes Investig. 2016 Apr;7(Suppl 1):38-43.

102. Javed K, Fairweather SJ. Amino acid transporters in the regulation of insulin secretion and signalling. Biochem Soc Trans. 2019 Apr 1;47(2):571-90. 
103. Adam J, Ramracheya R, Chibalina MV, Ternette N, Hamilton A, Tarasov Al, et al. Fumarate Hydratase Deletion in Pancreatic $\beta$ Cells Leads to Progressive Diabetes. Cell Rep. 2017 Sep 26;20(13):3135-48.

104. Eguchi N, Vaziri ND, Dafoe DC, Ichii H. The Role of Oxidative Stress in Pancreatic $\beta$ Cell Dysfunction in Diabetes. Int J Mol Sci. 2021 Jan;22(4):1509.

105. Robertson RP, Harmon JS. Pancreatic islet $\beta$-cell and oxidative stress: The importance of glutathione peroxidase. FEBS Lett. 2007 Jul 31;581(19):3743-8.

106. Zhang J, An H, Ni K, Chen B, Li H, Li Y, et al. Glutathione prevents chronic oscillating glucose intake-induced $\beta$-cell dedifferentiation and failure. Cell Death Dis. $2019 \mathrm{Apr}$ $11 ; 10(4): 1-13$.

107. Lacraz G, Figeac F, Movassat J, Kassis N, Coulaud J, Galinier A, et al. Diabetic $\beta$-Cells Can Achieve Self-Protection against Oxidative Stress through an Adaptive Up-Regulation of Their Antioxidant Defenses. PLOS ONE. 2009 Aug 5;4(8):e6500.

108. Zhang Z, Liew CW, Handy DE, Zhang Y, Leopold JA, Hu J, et al. High glucose inhibits glucose-6-phosphate dehydrogenase, leading to increased oxidative stress and $\beta$-cell apoptosis. FASEB J. 2010 May;24(5):1497-505.

109. Salvemini F, Franzé A, lervolino A, Filosa S, Salzano S, Ursini MV. Enhanced glutathione levels and oxidoresistance mediated by increased glucose-6-phosphate dehydrogenase expression. J Biol Chem. 1999 Jan 29;274(5):2750-7.

110. Pandolfi PP, Sonati F, Rivi R, Mason P, Grosveld F, Luzzatto L. Targeted disruption of the housekeeping gene encoding glucose 6-phosphate dehydrogenase (G6PD): G6PD is dispensable for pentose synthesis but essential for defense against oxidative stress. EMBO J. 1995 Nov 1;14(21):5209-15.

111. Mullarky E, Cantley LC. Diverting Glycolysis to Combat Oxidative Stress. In: Nakao K, Minato N, Uemoto S, editors. Innovative Medicine: Basic Research and Development [Internet]. Tokyo: Springer; 2015 [cited 2021 Aug 9]. Available from: http://www.ncbi.nlm.nih.gov/books/NBK500351/

112. PubChem. P(1),P(5)-Bis(5'-adenosyl) pentaphosphate(5-) [Internet]. [cited 2021 Aug 9]. Available from: https://pubchem.ncbi.nlm.nih.gov/compound/45479437 